\title{
Perioperative Monitoring in Liver Transplant Patients
}

\author{
Shweta Singh, Vaibhav Nasa, Manish Tandon \\ Dept. of Anesthesiology and Critical Care, Institute of Liver and Biliary Sciences, D1 Vasant Kunj, New Delhi 110070, India
}

\begin{abstract}
Liver transplant (LT) is a major surgical undertaking involving major fluid shifts, hemodynamic instability and metabolic derangements in a patient with preexisting liver failure and multisystemic derangements. Monitoring and organ support initiated in the preoperative phase is continued intraoperatively and into the postoperative phase to ensure an optimal outcome. As cardiovascular events are the leading cause of non-graft related death among LT recipients, major emphasis is placed on cardiovascular monitoring. The other essential monitoring are the continuous assessment of coagulapathy, extent of metabolic derangements, dyselectrolytemis and intracranial pressure monitoring in patients with fulminant hepatic failure. The type and extent of monitoring differs with need according to preexisting child status of the patient and the extent of systemic derangements. It also varies among transplant centers and is mainly determined by individual or institutional practices. ( $\mathrm{J}$ CuIN Exp Hepatol 2012;2:271-278)
\end{abstract}

$\mathrm{L}$ iver transplant (LT) has become a feasible treatment option for acute as well as chronic end stage liver disease (ESLD), irresectable liver malignancies and also for several metabolic abnormalities. Liver diseases, necessitating LT can either be acute or chronic. Each disease entity presents unique features as well as important differences.

While cirrhosis is a slow and insidious liver dysfunction, acute liver failure (ALF) presents with liver dysfunction, coagulopathy and hepatic encephalopathy occurring within days or weeks, often leading to a life-threatening multisystem illness and a rise in intracranial pressure (ICP). Severe hemodynamic, hematological and metabolic abnormalities are common.

Keywords: liver transplant, ALF, cirrhosis, coagulopathy, intracranial pressure monitoring

Received: 13.3.2012; Accepted: 26.6.2012; Available online: 25.8.2012

Address for correspondence: Shweta Singh, Associate Professor, Dept. of Anesthesiology and Critical Care, Institute of Liver and Biliary Sciences, D1 Vasant Kunj, New Delhi 110070, India. Tel.: +91 9810625177

E-mail: drshwetasingh29@gmail.com

Abbreviations: LT: liver transplant; ESLD: end stage liver disease; ALF: acute liver failure; ICP: intracranial pressure; CO: cardiac output; SVR: systemic vascular resistance; PAC: pulmonary artery catheter; CVP: central venous pressure; PAOP: pulmonary arterial occlusion pressure; TDCO: thermodilution principle; CCO: continuous CO; mPAP: mean pulmonary artery pressure; RVEDV: right ventricular end-diastolic volume; SV: stroke volume; EVLWI: extra vascular lung water index; CI: cardiac index; TEE: transesophageal echocardiography; CCTs: conventional coagulation tests; PT: prothrombin time; APTT: activated partial thromboplastin time; TEG: thrombelastography; ROTEM: rotation thrombelastometry; TEG: thrombelastography; MA: maximum amplitude; CL: clot lysis; ACT: activated clotting time; CR: clot rate; PF: platelet function; ICP: intracranial pressure; ICH: intracranial hypertension; TCD: transcranial Doppler; ONSD: optic nerve sheath diameter; PI: pulsatility index; ICG: indocyanine green; ARDS: acute respiratory distress syndrome

http://dx.doi.org/10.1016/j.jceh.2012.06.003
LT is a major surgical undertaking involving major hemodynamic shifts and metabolic derangements necessitating extensive monitoring and support of all organ systems. It is now becoming increasingly possible to offer transplant to sicker patients with multiple co-morbidities and organ dysfunction, as monitoring and organ support initiated in the preoperative phase is continued intraoperatively and into the postoperative phase to ensure an optimal outcome.

\section{HEMODYNAMIC MONITORING}

Cardiorespiratory failure has been identified as the commonest cause for ICU readmission after LT and cardiovascular events are the leading cause of non-graft related death among LT recipients. ${ }^{1}$ Major emphasis is therefore placed on cardiovascular monitoring during perioperative care of these patients.

Patients with cirrhosis and portal hypertension have a hyperdynamic circulatory state, with high cardiac output (CO) and a low systemic vascular resistance (SVR). ${ }^{2}$ There is systemic vasodilatation and marked increase in splanchnic capacitance. As a result, the central blood volume is significantly reduced. Due to this relative hypovolemia and major fluid shifts which occur during surgery, there is a need for a reliable hemodynamic monitoring tool for fluid management, so as to maintain the delicate balance between optimizing preload and avoiding pulmonary edema. ${ }^{3}$

Standard hemodynamic monitoring used routinely during adult LT includes continuous 5 lead ECG, invasive arterial pressure and cardiac output (CO) monitoring. Often both the radial arteries or a radial and a femoral artery are cannulated due to the need for frequent blood sampling and to enable continuous invasive blood pressure monitoring during the long surgery. Femoral arterial catheter is preferred over radial artery catheter by many, since 
central aortic pressure monitoring is considered more accurate especially at times of hemodynamic instability. Besides blood pressure monitoring using the radial artery is known to get affected by rib cage retraction causing subclavian artery compression. ${ }^{4}$

Changes in arterial and venous tone, intravascular volume, ventricular performance, peripheral vascular reactivity, core body temperature and changes in blood rheology make moment-to-moment assessment of cardiovascular status difficult. Clinical parameters like heart rate, blood pressure, blood loss and urine output are insufficient to direct fluid therapy for a recipient of LT. More detailed and accurate measurements are therefore needed for assessing cardiac preload, directing volume replacement and optimizing $\mathrm{CO}^{5}$

Traditionally, pulmonary artery catheter (PAC) has been used for invasive hemodynamic monitoring during LT. Cardiac filling pressures, namely central venous pressure (CVP) and pulmonary arterial occlusion pressure (PAOP) measured using the PAC serve as a guide to right and left heart preload respectively. Till recently these cardiac filling pressures were widely used to guide fluid therapy, but recent studies have shown that these pressure-derived parameters, which are indirect indicators of ventricular filling volumes, ${ }^{6,7}$ have little positive predictive value in improving hemodynamics or tissue perfusion. ${ }^{8}$

The PAC was initially used only to measure intracardiac pressures, but $\mathrm{CO}$ measurement using the thermodilution principle (TDCO) has now become an integral function of the PAC. Advances in technology have allowed for the development of continuous $\mathrm{CO}$ (CCO) monitoring by incorporating a heating coil within the PAC (CCOmbo/Vigilance, Edwards Lifesciences LLC, Irvine, CA; Opti Q CCO/Q-vue, Abbott Critical Care Systems, Mountain View, CA). This obviates the need for bolus injections and provides average $\mathrm{CO}$ over time compared with intermittent bolus techniques ${ }^{9}$ and still allows for monitoring of CVP, mean pulmonary artery pressure (mPAP) and PAOP. Advances in computation techniques have resulted in development of algorithms to calculate the global end-diastolic volume and the right ventricular end-diastolic (RVEDV) and end-systolic volumes, thereby facilitating better estimation of intravascular blood volume. ${ }^{10} \mathrm{RVEDV}$ is a valuable index of cardiac preload and is a more sensitive indicator of intravascular volume when compared with CVP and PAOP. ${ }^{11,12}$ The PAC however, might be inaccurate if it is not positioned correctly and may not reflect changes in intravascular volume rapidly enough. With reported hazards of PAC insertion like ventricular arrhythmias, ${ }^{13}$ and due to availability of less invasive monitoring tools, the use of PAC is declining. It is now increasingly reserved, for those cases where there is a suspicion of porto pulmonary hypertension, ${ }^{14}$ as severe pulmonary hypertension (mPAP > 45) is associated with high perioperative mortality and, if not successfully treated, is a contraindication to $\mathrm{LT}^{15}$
Standard hemodynamic monitoring, such as arterial pressure monitoring, can also be extended for the assessment of $\mathrm{CO}$, preload and afterload. A number of such devices are commercially available which provide a continuous estimate of $\mathrm{CO}$ through analysis of the shape of the arterial pulse wave from a peripherally placed arterial catheter. This technique helps measure stroke volume (SV) and CO on a beat-to-beat basis and helps assess requirement of therapies such as fluid challenge and/or inotropes. ${ }^{16}$

The PiCCO system (Pulsion Medical System; Munich, Germany) is a commercially available, continuous CO monitor in which a femoral arterial catheter with a thermistor in its wall analyses the pulse contour. The working principle is based on an algorithm which analyzes the shape of the arterial pressure waveform and computes the pulsatile systolic area. Beat-to-beat calculations are averaged over 30-s cycles and are displayed as a numerical value providing information regarding the patient's preload (intrathoracic blood volume, ITBVI), afterload, myocardial contractility, $\mathrm{CO}$ and extra vascular lung water index (EVLWI). ${ }^{17}$ ITBVI has shown a strong correlation with cardiac index (CI) as opposed to filling pressures in cirrhotics. It is regarded as a more reliable preload index during different phases of LT like during IVC clamping, graft reperfusion, bleeding and surgical manipulations. ${ }^{18}$ The device is initially calibrated by TDCO, using cold saline injection via central venous catheter and subsequent detection by the thermistor in the femoral arterial catheter. Beat-to-beat calculations are averaged over 30-s cycles and displayed as a numerical value. CCO assessed by PICCO and by TDCO have been found to be comparable. The monitor however, needs to be recalibrated if the SVR changes markedly. ${ }^{19,20}$

Other popular commercial equipment that make use of pulse power analysis include the LiDCO plus (LiDCO Ltd; Cambridge, UK) and the FloTrac/Vigileo (Edwards Lifesciences LLC; Irvine, CA). Both devices use proprietary algorithms to derive $\mathrm{CO}$ and like PiCCO, both display pulse pressure variation and stroke volume variation, which are indicative of intravascular volume status. ${ }^{21}$ Flo-Trac/Vigileo derives CO from the arterial waveform in conjunction with patient demographic data, without the need for an independent method of calibration. CO can be measured directly from a conventional arterial line attached to the sensor. ${ }^{22}$ The LiDCO system needs to be calibrated using lithium dye. However, its advantage over other pulse contour derived methods is that it provides beat to beat changes in $\mathrm{CO}$, while the Vigileo computes and displays SV values every 20 s. The values obtained from LiDCO and the FloTrac have been compared with PAC and found to be comparable. ${ }^{23}$

More recently transesophageal echocardiography (TEE) has become an essential perioperative diagnostic and monitoring tool. ${ }^{24}$ It provides direct visualization of the function and volume status of the heart. It allows quick 
assessment of the changes in contractility and rapid diagnosis of ventricular dilatation and failure. ${ }^{25}$ During times of hemodynamic instability, TEE can help with immediate diagnosis of air or thromboembolus. $\mathrm{CO}$ can be derived by measurement of flow across a cardiac valve, left ventricular outflow tract, or the flow in the main pulmonary artery. The functional utility of TEE, is however limited as a hemodynamic tool during LDLT due to the fear of rupture of esophageal varices ${ }^{26}$ but is considered a reliable, hemodynamic monitoring tool where there is adequate operator experience.

The preferred hemodynamic monitoring tool remains controversial because of lack of evidence indicating a difference in patient outcomes. Schumann ${ }^{27}$ surveyed 62 transplant centers in the United States and found that PACs were used in $30 \%$ of the centers and that TEE was used in $11.3 \%$. In summary, the type of monitoring differs among transplant centers and is mainly determined by individual or institutional practice. In most centers, an arterial line is placed which is extended for $\mathrm{CO}$ monitoring using either Flotrac, LiDCO or PICCO, depending on individual preferences. The PAC is usually reserved for cases with suspected PHT and the TEE is used as per availability of expertise. ${ }^{28}$

\section{COAGULATION MONITORING}

Patients with liver disease are at increased risk of both thrombosis as well as hemorrhage, ${ }^{29}$ In patients with cirrhosis of liver and ESLD there occur abnormalities of both procoagulants (e.g. vWF, Factor VIII) and anticoagulants (e.g. protein C, antithrombin and tissue factor pathway inhibitor). LT has been associated with major blood $\operatorname{loss}^{30}$ and need for transfusion of fairly large amounts of allogenic blood products.

During LT, dissection in the presence of coagulopathy through the fragile and dilated collaterals leads to blood loss. Transfusion needs in these patients may vary between 2 and 13 packed RBC units during liver transplant to much more. ${ }^{31,32}$

The inherent risks of use of blood products, along with scarcity of these resources, makes it important that these be used judiciously. Studies have linked intraoperative transfusions with significant decrease in 1 -year survival ${ }^{33}$ and the amount of blood product administered intraoperatively has been a significant predictor of ICU readmission. There has been a consistent decline in transfusion requirements over the years with refinement of surgical technique and revision in transfusion protocols.

The evaluation and correction of coagulopathy is an essential part of the care of the liver recipient in the perioperative period. It includes diagnosing potential causes of hemorrhage, prediction of risk of bleeding and appropriate hemostatic therapy. Close observation of the surgical field, suctions, communication with the surgical team along with monitoring of coagulation indices in the operating room are a crucial part of management. Traditionally, coagulation has been monitored by Conventional coagulation tests $(\mathrm{CCTs})^{34}$ which include Prothrombin time (PT), activated partial thromboplastin time (APTT), platelet count and plasma fibrinogen levels. Each of these measures a different aspect of the clotting process but even when put together, they may not provide a complete picture of the coagulation status and have a dubious role in guiding correction of coagulopathy prior to interventions.

Anesthesiologists and intensivists managing liver transplant patients usually use a combination of coagulation studies to guide blood product replacement therapy during LT. These include CCTs with point of care (POC) devices which assesses the viscoelastic properties of whole blood, like thrombelastography (TEG), rotation thrombelastometry (ROTEM) and Sonoclot to overcome the limitations of CCTs. ${ }^{35}$ These tests are repeated frequently during the surgery for correction of coagulopathy and reduce blood loss and transfusions.

Thrombelastography (TEG) which measures the elastic property of fibrin from its formation to complete dissolution is widely used to monitor coagulation status. The importance of TEG during LT as a test of whole-blood coagulation, has been established in literature. ${ }^{36}$ The test allows for in vivo interactions between platelets, red blood cells and other coagulation factors and clot development is displayed in real-time.

Thrombelastometry (ROTEM) is another POC test. It is a variation of TEG and is based on measuring shear-elastic modules during clot formation in whole blood. TEG and ROTEM both measure and graphically display the changes in viscoelasticity of blood at all stages of the developing and resolving clot, i.e., the time until initial fibrin formation (reaction time $[R]$, the kinetics of fibrin formation and clot development (angle $[\alpha]$ ), the ultimate strength and stability of the fibrin clot (maximum amplitude $[\mathrm{MA}]$ ), and clot lysis (fibrinolysis [CL]). ${ }^{37}$ (Figure 1) TEG and ROTEM are both fibrinolysis sensitive assays and allow for diagnosis of hyperfibrinolysis in bleeding patients $^{38,39}$ and hence enable directed therapy. Literature shows that use of TEG and ROTEM in transfusion algorithms has led to reduced number of blood products transfused..$^{40}$ A survey undertaken in the United States revealed that TEG is being used only by a third of all

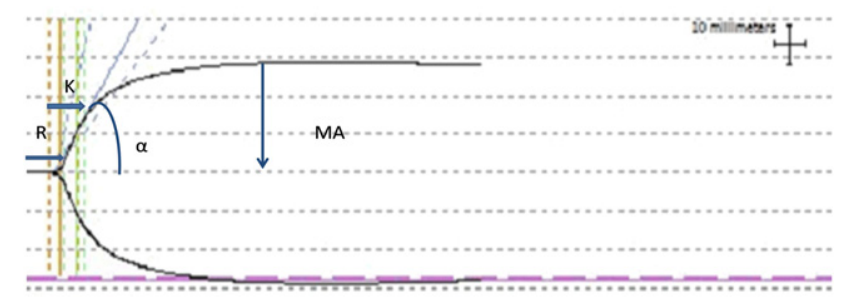

Figure 1 Thrombelastograph (TEG) trace: R: reaction time; K: kinetics; $\alpha$ : slope between R \& K; MA: maximum amplitude; CL: clot lysis. 
programs involved with $\mathrm{LT}$, while pediatric LT units rely more on CCT for coagulopathycorrection. ${ }^{27}$

ROTEM has been successfully used primarily in Europe. The reason for this disparity could be institutional availability and protocols.

The Sonoclot measurements are based on detection of viscoelastic changes of whole blood or plasma. The Sonoclot Analyzer measures the kinetics of fibrin formation and clot development and provides information on the entire hemostasis process both as a qualitative graph, known as Sonoclot Signature and the quantitative values e.g. CR (the maximum slope of the Sonoclot Signature during initial fibrin polymerization and clot development), activated clotting time (ACT), clot rate (CR), and the platelet function (PF). The ACT is the time from activation of sample until the beginning of fibrin formation (Figure 2).

However, the Sonoclot Analyzer has been criticized because its results were influenced by age, gender and platelet count $^{41}$ and it has shown poor reproducibility of measured variables, especially $\mathrm{CR}$ and $\mathrm{PF}^{42}$ Others have found the Sonoclot Analyzer to be valuable in cardiac surgical procedures ${ }^{43,44}$ and it has demonstrated a precision close to that of TEG. In more recent studies, test variability of ACT values determined by Sonoclot, were comparable in LT and has been found to be useful in the perioperative coagulation management. ${ }^{45}$

\section{METABOLIC}

\section{Glycemic Control}

The liver plays a central role in regulation of whole-body metabolism. Liver disease therefore can lead to major alterations in glucose, lipid and protein metabolism. Patients with cirrhosis have a peculiar endocrine profile with impaired glucose homeostasis. Serum insulin concentration in these patients is elevated by at least 2-folds while the glucagon concentration is increased by 5 -folds. This occurs more due to

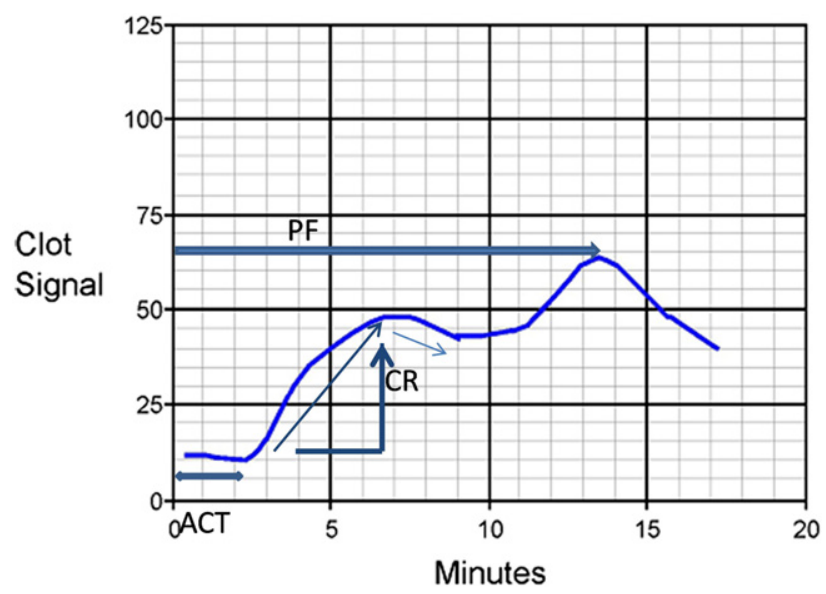

Figure 2 Sonoclot Signature: ACT: activated clotting time; CR: clot rate; PF: platelet function. augmented secretions than due to delayed clearance by the diseased liver. ${ }^{46}$ Despite normal gluconeogesis, there is hyperglycemia with hyperinsulinemia on account of impaired peripheral glucose oxidation due to insulin resistance. ${ }^{47}$

Glucose metabolism further worsens during LT. Hyperglycemia during liver transplant surgery develops following steroid therapy during the reperfusion period, and is aggravated further due to glycogenolysis by the newly transplanted liver, decreased peripheral glucose utilization and insulin resistance. ${ }^{48}$ Poor blood sugar control in liver transplant recipients has been linked to increased risk of liver allograft rejection, surgical site infection and increased mortality. ${ }^{49,50}$ While normal glucose metabolism, (with increased insulin requirement), could be a sign of a well functioning allograft post transplant. Hypoglycemia can be an ominous sign of compromised liver recovery. ${ }^{51}$ Hence frequent plasma glucose monitoring and blood sugar control become important. Although tight blood sugar control has been linked to improved outcomes (between 80 and $120 \mathrm{mg} / \mathrm{dL}$ ), this is associated with increased risk of hypoglycemia in the presence of reduced glucose reserve. A modest target of blood glucose of $\leq 150 \mathrm{mg} / \mathrm{dL}$ is therefore advised. ${ }^{52}$

\section{Electrolytes}

Dyselectrolytemias frequently accompany ALF and ESLD, especially when complicated by renal failure. Electrolyte imbalance in these settings may be particularly deleterious. Frequent monitoring of serum electrolyte concentrations throughout the perioperative period and correction of abnormalities is a routine practice.

Hyponatremia is commonly seen in patients with cirrhosis. Increased plasma renin activity along with hyperaldosteronism leads to $\mathrm{Na}^{+}$and water retention. However, free water retention exceeds $\mathrm{Na}^{+}$accumulation thereby causing dilutional hyponatremia. Hyponatremia may exacerbate cerebral edema in patients of ALF. Therapeutic measures adopted include free water restriction, and use of high caloric hyperosmolar enteral feeds or concentrated glucose infusions.

The risk of osmotic demyelination is lower in patients with ALF because of shorter duration of the disease and is treated using hypertonic saline. Careful calculation of rate of correction of plasma sodium levels is done to prevent central pontine myelinolysis. ${ }^{53,54}$

Serum potassium levels are independent predictors of death after liver transplantation. ${ }^{55}$ Serum $\mathrm{K}^{+}$concentrations are known to vary during different phases of liver transplantation. Hypokalemia is often secondary to diuretic therapy whereas hyperkalemia is characteristically seen following organ reperfusion. Retrospective studies have identified several variables associated with clinically significant hyperkalemia which include higher baseline potassium, amount of red cells transfused, organ recovery 
after cardiac death, longer warm ischemia time, and longer donor hospital stay. ${ }^{56}$ Hyperkalemia can also be caused by the use of potassium-sparing diuretics, metabolic acidosis causing exchange of intracellular potassium for extra- cellular $\mathrm{H}^{+}$, renal failure or secondary to hepato-renal syndrome.

Serum $\mathrm{Ca}^{2+}$ concentration is usually decreased by chelation with citrate following transfusions of blood products. In the absence of a healthy liver, citrate is not rapidly metabolized and ionized calcium may decrease precipitously during rapid intraoperative transfusion or during the anhepatic stage. ${ }^{57}$

Patients with ESLD usually also have hypomagnesemia. Multiple factors which contribute to hypomagnesemia are decreased dietary intake, decreased gut absorption and increased excretion due to laxatives and diuretics used to treat hepatic encephalopathy and ascites respectively. ${ }^{58}$ Hypomagnesemia further worsens during liver transplantation due to massive blood transfusion since ionized magnesium is chelated in a manner similar to chelation of ionized calcium by citrate. ${ }^{59}$ Hypomagnesemia is known to contribute to cardiac dysrhythmias ${ }^{60}$ and also has a crucial role in the coagulation cascade. ${ }^{61}$ Magnesium levels are therefore frequently monitored and corrected in the perioperative setting. ${ }^{58}$

\section{Acid-base Disorders}

Acid-base abnormalities are common in patients posted for liver transplant. The commonest acid-base disturbance in ESLD being primary respiratory alkalosis, ${ }^{62}$ mediated by a central nervous system mechanism. The C-fibers located in alveolar epithelium, near pulmonary capillaries respond to increased interstitial lung water by signaling the respiratory center to cause rapid shallow breathing. Metabolic alkalosis if present is usually iatrogenic, induced by diuretics $^{63}$ or due to the protracted vomiting. Patients in fulminant hepatic failure and hepato-renal syndrome may develop metabolic acidosis due to the inability of the liver to clear lactate and inability of the dysfunctional kidney to retain bicarbonate. Although metabolic acidosis is an uncommon acid-base abnormality in cirrhosis, it is the most ominous.

Patients with normal acid-base balance at the outset of surgery also develop biphasic acid-base disturbance, characterized by initial metabolic acidosis followed by protracted metabolic alkalosis during LT. ${ }^{64}$ The severity of metabolic acidosis parallels the accumulation of lactic acid, indicating that the major etiology is lactic acidosis. Loss of base equivalents starts during the dissection phase and accelerates during the anhepatic stage ${ }^{65} \mathrm{~A}$ further rise in plasma lactate concentration occurs immediately after graft reperfusion. Maximal acidosis and base deficit is therefore seen in the first several minutes after graft reperfusion and the main consequence of metabolic acidosis is myocardial depression. Plasma lactate concentrations are closely monitored during the surgery and following graft reperfusion and are also followed into the early postoperative period, for assessing the adequacy of graft function.

Early recognition of metabolic disturbances and their subsequent treatment improves outcome and decreases perioperative mortality. Monitoring of rapidly changing metabolic disturbances is therefore an inevitable part of rational intraoperative management in LT.

\section{Temperature}

Continuous temperature monitoring and use of devices to maintain normothermia is a part of standard management of patients for LT throughout the perioperative period. Negative effects of hypothermia on wound healing and on coagulation are well known even in the presence of otherwise normal plasma clotting factor concentrations. Mild hypothermia, on the other hand, has been suggested as an adjunct therapy for the treatment of raised intracranial pressure (ICP) to decrease brain metabolism and reduce oxidative stress in ALF.

\section{INTRACRANIAL PRESSURE MONITORING}

Cerebral edema leading to intracranial hypertension (ICH) is a major cause of morbidity and mortality in patients with ALF. ${ }^{66}$ ICP monitoring therefore is proposed for early diagnosis and management of ICH. It helps guide medical treatment and select patients with better neurologic prognosis for $\mathrm{LT}^{67}$ Additionally it has been shown that ICP tends to vary significantly intraoperatively during the surgery. It initially increases during the dissection phase, then decreases in the anhepatic phase, and again increases during the reperfusion phase. ${ }^{68}$ The main concern with placement of ICP monitors is the risk of intracranial hemorrhage. In a survey across medical centers in the United States in the early 1990s, an overall prevalence of intracranial bleeding of $20 \%$ was noted with the procedure. ${ }^{69}$

The use of noninvasive methods like Transcranial Doppler (TCD) and Optic Nerve Sheath Diameter (ONSD) is becoming popular as they avoid the serious complications of invasive ICP monitoring. TCD applies ultrasound to measure blood flow velocity in the middle cerebral artery. The difference between systolic and diastolic flow velocity, divided by the mean flow velocity, is called the pulsatility index (PI) which correlates well with invasively measured ICP. ${ }^{70}$

In ONSD measurement technique, the changes in the diameter of the nerve sheath are visualized using transocular ultrasound. The optic nerve which is a part of the central nervous system is surrounded by the dural sheath. With rise in ICP, the sheath expands. Several studies have investigated and found a correlation between the nerve sheath diameter and invasively measured ICP. ${ }^{71}$

Both these techniques of ICP monitoring, require training and have inter-observer variance. These techniques are not accurate enough to be used as a replacement for 
invasive ICP measuring methods. They can, however, distinguish between normal and raised $(>20 \mathrm{mmHg}$ ) ICP and can be used as a repeatable bedside screening modality in the coagulopathic patient of liver failure (Table 1).

\section{Monitoring in Post-Operative Period}

The principle behind institution of monitoring facilities for liver transplant patients in the postoperative period, is to provide the necessary support for maintaining optimal function of all organ systems. All intraoperative modalities of monitoring and medication are continued into the ICU. This would help provide a favorable milieu for the new liver to start functioning and at the same time would help in following the course of graft functioning.

Early weaning from mechanical ventilation after LT is possible in select patients with preoperative Child A status and an uneventful intraoperative course. This not only lowers the risk of ventilator associated pneumonia but also improves the splanchnic and liver blood flow and reduces hepatic venous congestion. ${ }^{72}$ However fast tracking following LT is not a routine practice due to the underlying liver disease with metabolic derangements, prolonged surgery with major fluid shifts, blood loss, hemodynamic instability; and prolonged warm and cold ischemia times.

The key areas of focus are hemodynamic stabilization with optimal fluid therapy, preservation of kidney function, prevention of graft rejection and infection prophylaxis. Subsequent ICU course is dependant on uptake of graft. Graft function is assessed at regular intervals by serial blood gas analysis, liver function tests and bedside ultrasound Doppler of hepatic vasculature. Improvement in metabolic acidosis, decline in blood lactate levels, and spontaneous improvement in INR are indicative of adequate graft uptake. Static and dynamic liver function tests are used to track graft function. Static tests of liver function include serum levels of liver enzymes, bilirubin and tests of synthetic function including serum level of albumin and coagulation factors. The dynamic liver function tests express the ability of the liver to metabolize or eliminate defined substances. The indocyanine green (ICG) clearance test is used in some centers routinely to quantify the functional activity of the graft. ICG is a water-soluble dye which can be used bedside or intraoperatively. Its removal from the blood depends on liver blood flow, parenchymal cellular function and biliary excretion. ${ }^{28}$

Ultrasound assessment of blood flow along with ICG clearance is currently regarded as the most valuable bedside liver function test in the perioperative period after LT. $^{28}$

Accurate monitoring of fluid intake and output is critical in the immediate postoperative period. Oliguria may be the earliest warning sign of renal dysfunction. This is supplemented by regular monitoring of kidney function tests. Intraabdominal drains are inspected for the nature and rate of blood and fluid loss. Various kinds of anastomotic problems may present in the early postoperative days with varying incidence. Hepatic artery thrombosis, with incidence of $4-12 \%,{ }^{73}$ can present as sudden deterioration in hemodynamics, Acute Respiratory Distress Syndrome (ARDS), severe coagulopathy, or sudden and marked elevation of transaminases.

Biochemical, hematological and microbiological monitoring is implemented depending on institutional protocols. Blood levels of immunosuppressants are measured to titrate and achieve recommended levels to avoid rejection and prevent drug toxicity. Initially more frequent monitoring is done, upto thrice a week and as blood levels stabilize the frequency is reduced.

Evaluation of the patient's mental status is also continually performed during the ICU stay as neurological complications are very common after LT. Clinical series have documented neurological disorders in $8.3-47 \%$ of all patients receiving liver transplantation. ${ }^{74}$ The most frequent complications are encephalopathy, brain hemorrhage, and seizures. Patients with neurologic symptoms prior to LT are at a higher risk for postoperative neurologic disturbances. Poor graft function or small for size may result in recurrence of hepatic encephalopathy. Patients transplanted for fulminant hepatic failure may need continuation of ICP monitoring to guide further management till clinical recovery of mentation occurs. Daily assessment of neurological status and muscle power is important for detection of focal and global

Table 1 Comparison of different ICP monitoring techniques.

\begin{tabular}{|c|c|c|c|c|c|}
\hline Technique & Accuracy & Incidence of infection & Incidence of bleeding & Cost per patient & Miscellaneous \\
\hline Invasive monitoring devices & High & Moderate to high & Low to moderate & High & Continuous bedside monitoring \\
\hline Transcranial Doppler & Low & None & None & Low & $\begin{array}{l}\text { Bedside monitoring; Inter observer } \\
\text { variability high }\end{array}$ \\
\hline Optic Nerve Sheath Diameter & Low & None & None & Low & $\begin{array}{l}\text { Bedside monitoring; Inter observer } \\
\text { variability high }\end{array}$ \\
\hline MRI/CT scan & Low & None & None & Low & Potential to be used for screening \\
\hline Fundoscopy & Low & None & None & Low & $\begin{array}{l}\text { Bedside monitoring; may fail to } \\
\text { detect sudden rise in ICP }\end{array}$ \\
\hline
\end{tabular}


deficits that could lead to the suspicion of stroke or embolism and the need for imaging.

\section{CONCLUSION}

The monitoring strategy of liver transplant centers is not uniform and is primarily governed by local practice patterns and patient outcome. Recent cost benefit analysis of resource utilization has changed existing practices. These have helped weigh the benefit of using one monitoring modality over the other. To bring uniformity in the existing practices reflects the need for randomized controlled trials, for drafting consensus protocols for optimum outcome and resource utilization.

\section{CONFLICTS OF INTEREST}

All authors have none to declare.

\section{REFERENCES}

1. Levy MF, Greene L, Ramsay MA, et al. Readmission to the intensive care unit after liver transplantation. Crit Care Med. 2001;29:18-24.

2. Henriksen JH, Bendtsen F, Sorensen TIA, et al. Reduced central blood volume in cirrhosis. Gastroenterology. 1989;97:15061513.

3. Liu H, Gaskari SA, Lee SS. Cardiac and vascular changes in cirrhosis: pathogenic mechanisms. World J Gastroenterol. 2006;12: 837-842.

4. Dulitz MG, De Wolf AM, Wong $\mathrm{H}$, et al. Compression of brachial plexus during right lobe liver donation as a cause of brachial plexus injury: a case report. Liver Transplant. 2005;11:233-235.

5. Moller S, Henriksen JH. Cardiovascular complications in cirrhosis. Gut. 2008;57:268-278.

6. De Wolf A. Hemodynamic monitoring during orthotopic liver transplantation. Transpl Proc. 1993;25:1863.

7. De Wolf AM, Begliomini B, Gasior TA, et al. Right ventricular function during orthotopic liver transplantation. Anesth Analg. 1993;76:562.

8. Kumar A, Anel R, Bunnell E, et al. Pulmonary artery occlusion pressure and central venous pressure fail to predict ventricular filling volume, cardiac performance, or the response to volume infusion in normal subjects. Crit Care Med. 2004;32:691.

9. Greim CA, Roewer N, Thiel H, et al. Continuous cardiac output monitoring during adult liver transplantation: thermal filament technique versus bolus thermodilution. Anesth Analg. 1997;85:483-488.

10. Della Rocca G, Costa MG, Feltracco P, et al. Continuous right ventricular end diastolic volume and right ventricular ejection fraction during liver transplantation: a multi center study. Liver Transpl. 2008;14:327-332.

11. Cheatham ML, Nelson LD, Chang MC, Safesak K. Right ventricular end diastolic volume index as a predictor of preload status in patients on positive end expiratory pressure. Crit Care Med. 1998;26:1801-1806.

12. Costa MG, Chiarandini P, Della Rocca G. Hemodynamics during liver transplantation. Transpl Proc. 2007;39:1871-1873.

13. Gwak MS, Kim JA, Kim GS, et al. Incidence of severe ventricular arrhythmias during pulmonary artery catheterization in liver allograft recipients. Liver Transpl. 2007;13:1451-1454.

14. Wiener RS, Welch HG. Trends in the use of the pulmonary artery catheter in the United States, 1993-2004. JAMA. 2007;298: 423-429.
15. Murray KF, Carithers RL. AASLD practice guidelines: evaluation of the patient for liver transplantation. Hepatology. 2005;41(6):1407-1432.

16. Della Rocca G, Brondani Anita, Costa Maria Gabriella. Intraoperative hemodynamic monitoring during organ transplantation: what is new? Curr Opin Organ Transpl. 2009;14:291-296.

17. Diaz S, Perez-Pena J, Sanz J, et al. Haemodynamic monitoring and liver function evaluation by pulsion cold system Z-201 (PCS) during orthotopic liver transplantation. Clin Transplan. 2003;17:47.

18. DellaRocca G, Costa MG, Coccia C, et al. Preload and haemodynamic assessment during liver transplantation: a comparison between the pulmonary artery catheter and transpulmonary indicator dilution techniques. Eur J Anaesthesiol. 2002;19:868-887.

19. Della Rocca G, Costa MG, Coccia C, et al. Cardiac output monitoring: aortic transpulmonary thermodilution and pulse contour analysis agree with standard thermodilution methods in patients undergoing lung transplantation. Can J Anesth. 2003;50:707-711.

20. Della Rocca G, Costa MG, Pompei L, et al. Continuous and intermittent cardiac output measurement: pulmonary artery catheter versus aortic transpulmonary technique. Br J Anaesth. 2002;88:350-356.

21. Button D, Weibel L, Reuthebuch O, Genoni M, Zollinger A, Hofer CK. Clinical evaluation of the FloTrac/Vigileo ${ }^{\mathrm{TM}}$ system and two established continuous cardiac output monitoring devices in patients undergoing cardiac surgery. Br J Anaesth. 2007;99:329-336.

22. Biais M, Nouette-Gaulain $\mathrm{K}$, Cottenceau V, et al. Cardiac output measurement in patients undergoing liver transplantation: pulmonary artery catheter versus uncalibrated arterial pressure waveform analysis. Anesth Analg. 2008;106:1480-1486.

23. Biancofiore G, Critchley LA, Lee A, et al. Evaluation of an uncalibrated arterial pulse contour cardiac output monitoring system in cirrhotic patients undergoing liver surgery. Br J Anaesth. 2009;102:47-54.

24. De Wolf AM, Aggarwal S. Monitoring preload during liver transplantation. Liver Transpl. 2008;14:268-269.

25. Burtenshaw AJ, Isaac JL. The role of trans-oesophageal echocardiography for perioperative cardiovascular monitoring during orthotopic liver transplantation. Liver Transpl. 2006;12:1577-1583.

26. Spier BJ, Larue SJ, Teelin TC, et al. Review of complications in a series of patients with known gastro-esophageal varices undergoing transesophageal echocardiography. J Am Soc Echocardiogr. 2009;22:401-403.

27. Schumann R. Intraoperative resource utilization in anesthesia for liver transplantation in the United States: a survey. Anesth Analg. 2003;97:21-28.

28. Krenn Claus-Georg, De Wolf Andre M. Current approach to intraoperative monitoring in liver transplantation. Curr Opin Organ Transpl. 2008;13:285-290.

29. Roberts LN, Patel RK, Roopen. Haemostasis and thrombosis in liver disease. Br J Haematol. 2009;148:507-521.

30. Yuasa T, Niwa N, Kimura S, et al. Intraoperative blood loss during liver transplantation: an analysis of 635 recipients at a single center. Transfusion. 2005;45:879.

31. de Boer MT, Molenaar IQ, Hendriks HG, et al. Minimizing blood loss in liver transplantation: progress through research and evolution of techniques. Dig Surg. 2005;22:265-275.

32. Ozier Y, Pessione F, Samain E, et al. French study group on blood transfusion in liver transplantation. Institutional variability in transfusion practice for liver transplantation. Anesth Analg. 2003; 97:671.

33. Butler P, Israel L, Nusbacher J, et al. Blood transfusion in liver transplantation. Transfusion. 1985;25:120.

34. Cacciarelli TV, Keeffe EB, Moore DH, et al. Effect of intraoperative blood transfusion on patient outcome in hepatic transplantation. Arch Surg. 1999;134:25.

35. Tripodi A, Mannucci PM. Abnormalities of hemostasis in chronic liver disease: Reappraisal of their clinical significance and need for clinical and laboratory research. J Hepatol. 2007;46:727. 
36. Massicotte L, Beaulieu D, Thibeault L, et al. Coagulation defects do not predict blood product requirements during liver transplantation. Transplantation. 2008;85:956-962.

37. Salooja N, Perry DJ. Thromboelastography. Blood Coag Fibrin. 2001;12:327-337.

38. Ganter MT, Hofer CF. Coagulation monitoring: current techniques and clinical use of viscoelastic point-of-care coagulation devices. Int Anesth Res Soc. 2008;106(5):1366-1375.

39. Roullet S, Pillot J, Freyburger G, et al. Rotation thromboelastometry detects thrombocytopenia and hypofibrinogenaemia during orthotopic liver transplantation. Br J Anaesth. 2010;104:422-428.

40. Coakley M, Reddy K, Mackie I, et al. Transfusion triggers in orthotopic liver transplantation: a comparison of the thromboelastometry analyzer, the thromboelastogram, and conventional coagulation tests. J Cardiothorac Vasc Anesth. 2006;20:548-553.

41. Horlocker TT, Schroeder DR. Effect of age, gender, and platelet counton Sonoclot coagulation analysis in patients undergoing orthopedic operations. Mayo Clin Proc. 1997;72:214-219.

42. Ekback G, Carlsson O, Schott U. Sonoclot coagulation analysis: a study of test variability. J Cardiothorac Vasc Anesth. 1999;13: 393-397.

43. MiyashitaT KuroM. Evaluation of platelet function by SonoClot analysis compared with other hemostatic variables in cardiac surgery. Anesth Analg. 1998;87:1228-1233.

44. Saleem A, Blifeld C, Saleh SA, et al. Viscoelastic measurement of clot formation: a new test of platelet function. Ann Clin Lab Sci. 1983;13:115-124.

45. Chapin JW, Becker GL, Hulbert BJ, et al. Comparison of thromboelastograph and Sonoclot coagulation analyzer for assessing coagulation status during orthotopic liver transplantation. Transpl Proc. 1989;21:3539.

46. Shangraw RE, Jahoor F. Mechanism of dichloroacetate-induced hypolactatemia in humans with or without cirrhosis. Metabolism. 2004;53:1087-1094.

47. Kruszynska YT, Home PD, McIntyre N. Relationship between insulin sensitivity, insulin secretion and glucose tolerance in cirrhosis. Hepatology. 1991;14:103-111.

48. Shmueli E, Walker M, Alberti G, et al. Normal splanchnic but impaired peripheral insulin-stimulated glucose uptake in cirrhosis. Hepatology. 1993;18:86-95.

49. Wallia A, Parikh ND, Molitch ME, et al. Posttransplant hyperglycemia is associated with increased risk of liver allograft rejection. Transplantation. 2010;89:222-226.

50. Park C, Hsu C, Neelakanta G, et al. Severe intraoperative hyperglycemia is independently associated with surgical site infection after liver transplantation. Transplantation. 2009;87:1031-1036.

51. Marik PE, Preiser JC. Towards understanding tight glycemic control in the ICU: a systematic review and meta-analysis. Chest. 2009;137:544-551.

52. Finfer S, Chittock DR, Su SY, et al. Intensive versus conventional glucose control in critically ill patients. N Engl J Med. 2009;360: 1283-1297.

53. Hackworth WA, Heuman DM, Sanyal AJ, et al. Effect of hyponatraemia on outcomes following orthotopic liver transplantation. Liver Int. 2009;29:1071-1077.

54. Yun BC, Kim WR, Benson JT, et al. Impact of pretransplant hyponatremia on outcome following liver transplantation. Hepatology. 2009;49:1610-1615.
55. Dawwas MF, Lewsey JD, Watson CJ, et al. The impact of serum potassium concentration on mortality after liver transplantation: a cohort multicenter study. Transplantation. 2009;88:402-410.

56. Xia VW, Ghobrial RM, Du B, et al. Predictors of hyperkalemia in the prereperfusion, early postreperfusion, and late post reperfusion periods during adult liver transplantation. Anesth Analg. 2007;105: 780-785.

57. Merritt WT. Metabolism and liver transplantation: review of perioperative issues. Liver Transpl. 2000;6(4 suppl. 1):S76-S84.

58. Diaz J, Acosta F, Parrilla P, et al. Serum ionized magnesium monitoring during orthotopic liver transplantation. Transplantation. 1996;61:835-837.

59. Ranasinghe DN, Mallett SV. Hypomagnesaemia, cardiac arrhythmias and orthotopic liver transplantation. Anaesthesia. 1994;49:403-405.

60. Scott VL, De Wolf AM, Kang Y, et al. lonized hypomagnesemia in patients undergoing orthotopic liver transplantation: a complication of citrate. Liver Transpl Surg. 1996 Sep;2(5):343-347.

61. Bennett MW, Webster NR, Sadek SA. Alterations in plasma magnesium concentrations during liver transplantation. Transplantation. 1993;56:859-861.

62. Moreau R, Hadengue A, Soupison T, et al. Arterial and mixed venous acid-base status in patients with cirrhosis. Influence of liver failure. Liver. 1993;13:20-24.

63. Shangraw RE. Acid-base balance. In: Miller RD, ed. Anesthesia. 5th ed. Philadelphia: Churchill Livingstone; 2000:1390-1413.

64. Shangraw RE, Robinson ST. Oxygen metabolism during liver transplantation: the effect of dichloroacetate. Anesth Analg. 1997;85: 746-752.

65. Shangraw RE, Winter R, Hromco J, et al. Amelioration of lactic acidosis with dichloroacetate during liver transplantation in humans. Anesthesiology. 1994;81:1127-1138.

66. Raschke RA, Curry SC, Rempe S, Gerkin R, et al. Results of a proto$\mathrm{col}$ for the management of patients with fulminant liver failure. Crit Care Med. 2008;36(8):2244-2248.

67. Polson J, Lee WM. The management of acute liver failure. Hepatology. 2005;41:1179-1197.

68. Lidofsky SD, Bass NM, Prager MC, et al. Intracranial pressure monitoring and liver transplantation for fulminant hepatic failure. Hepatology. 1992;16:1-7.

69. Blei AT, Olafsson S, Webster S, Levy R. Complications of intracranial pressure monitoring in fulminant hepatic failure. Lancet. 1993;341:157-158.

70. J. Bellner, B. Romner, P. Reinstrup, K. A. Kristiansson, E. Ryding, and L.Brandt. Transcranial Doppler sonography pulsatility index (PI) reflects intracranial pressure (ICP).

71. Geeraerts T, Launey Y, Martin L, et al. Ultrasonography of optic nerve sheath maybe useful for detecting raised intra cranial pressure after severe brain injury. Intensive Care Med. 2007;33(10): 1704-1711.

72. Gopal PB, Kapoor D, Raya Ravichandra, et al. Critical care issues in adult liver transplantation. Indian J Crit Care Med. 2009;13(3): 113-119.

73. Pastacaldi S, Teixeira R, Montalto P, Rolles K, Burroughs AK. Hepatic artery thrombosis after orthotopic liver transplantation: a review of nonsurgical causes. Liver Transpl. 2001;7:75-81.

74. Lewis MB, Howdle PD. Neurologic complications of liver transplantation in adults. Neurology. 2003;61:1174-1178. 